\title{
A multidimensional scaling analysis of 16 complex sounds
}

\author{
JAMES H. HOWARD, JR., and EUGENE B. SILVERMAN \\ The Catholic University of America, Washington, D. C. 20064
}

\begin{abstract}
The INDSCAL multidimensional scaling model was used to investigate the distinctive features involved in the perception of 16 complex nonspeech sounds. The signals differed along four physical dimensibns: fundamental frequency, waveform, formant frequency, and number of formants. Scaling results indicąted that subjects' similarity ratings could be accounted for by three psychological or perceptual dimensions. A statistically reliable correspondence was observed between these perceptual dimensions and the physical characteristics of fundamental frequency, waveform, and a combination of the two formant parameters. These results were further explored with Johnson's (1967) hierarchical clustering analysis. Large differences in featural saliency occurred in the group data with fundamental accounting for more variability than the remaining dimensions. Further analysis of individual subject data revealed large individual differences in featural saliency. These differences were related to past musical experience of the subject and to earlier findings using similar signals. It was concluded that (1) the INDSCAL model provides a useful method for the analysis of auditory perception in the nonspeech mode, and (2) featural saliency in such sounds is likely to be determined by an unspecified attentional mechanism.
\end{abstract}

The human ability to perceive complex sounds is of central importance as demonstrated by our continual reliance on speech as a medium of communication. Although the perception of speech sounds is clearly important, we readily perceive a variety of other complex sounds as well (e.g., the sounds of aircraft, birds, and musical instruments). While much research effort has been expended in an attempt to understand the perception of speech (e.g., Kavanagh \& Mattingly, 1972; Liberman, 1970), relatively little research has focused on the perception of other complex sounds (e.g., Massaro, 1972; Webster, Woodhead, \& Carpenter, 1973). This paper investigates the multidimensional structure underlying the perception of a specific set of 16 complex nonspeech sounds.

Auditory perception, as any cognitive process, will be constrained by both the information available to the subject (i.e., in the signal) and the processes applied to the information by the subject (Garner, 1974; Norman \& Bobrow, 1975). While both of these performance-limiting factors influence perception, the second, or process, factor is of primary psychological importance. What are the processes that underlie the perception of complex sounds? In general, research has indicated that auditory perception depends crucially on the ability of the subject to extract information about the presence of

This research was supported by contract No. N00014-75-C-0308 from the Office of Naval Research, Engineering Psychology Programs. Requests for reprints should be addressed to James $\mathbf{H}$. Howard. Jr., Department of Psychology. The Catholic University, Washington, D.C. 20064. The authors thank Bell Telephone Laboratories for providing copies of the INDSCAL and HICLUST programs. certain distinctive features in the acoustic signal (e.g., Mattingly, Liberman, Syrdal, \& Halwes, 1971; Webster et al., 1973). Consequently, an understanding of this "feature extraction" process is central to the understanding of auditory perception.

Since the assumed feature extraction process is not directly observable, the features involved in perception can only be inferred from performance data. For example, subjects could be asked to classify synthetically generated signals of varying physical specification (e.g., Mattingly et al., 1971). Here, the relation between the subject's response and the physical characteristics of the signals would be examined. A high correlation between the pattern of responding and the value of a particular physical characteristic would indicate that the physical dimension is important psychologically. Alternatively, the features underlying perception could be determined by subjecting the end result of the perceptual process-some prescribed perceptual response-to a multidimensional scaling analysis (e.g., Carroll \& Wish, 1974a; Shepard, Nerlove, \& Romney, 1972). The outcome of this analysis is a decomposition of the perceptual data into a set of $n$ orthogonal dimensions or features. Each stimulus can be represented as a point in the space defined by the $n$ features. For example, Shepard (1972) and Wish (Note 1) have scaled Miller and Nicely's (1955) perceptual confusion data for 16 consonant phonemes with impressive success. Similarly, Carterette \& Barnebey (Note 2) have undertaken an analysis of the features underlying human voice recognition with promising results.

Several studies have used the first of the above methods to examine the features involved in the 
perception of complex nonspeech sounds. In a recent study. Webster et al. (1973) required subjects to learn labels for a set of 16 complex sounds. The sounds varied along four physical dimensions: fundamental frequency. waveform. number of formants, and formant frequency. Their results indicated that subjects were more likely to confuse sounds differing in formant characteristics (i.e.. formant frequency and number of formants) than they were to confuse sounds differing in source characteristics (i.e., fundamental and waveform). They concluded that. while all four physical parameters were important psychologically, the relative importance or saliency of these features differed considerably, with fundamental frequency and waveform being more salient than formant frequency and number of formants.

The present study employs the INDSCAL multidimensional scaling procedure to analyze the distinctive features involved in the perception of complex sounds similar to those studied by Webster et al. (1973). In the study, subjects are asked to provide similarity ratings for all possible pairs of a set of 16 complex sounds. The INDSCAL model assumes that an individual subject's judgment of stimulus similarity is a decreasing linear function of the interstimulus distance (assuming a modified Euclidean metric) in a hypothetical underlying perceptual space (Carroll \& Chang, 1970). Subject proximity judgments are converted to estimated distances using Torgerson's (1958) additive constant estimation method (Carroll \& Wish, 1974b). It differs from most other metric scaling procedures in that information regarding individual subjects is preserved (cf. Shepard et al., 1972). The net outcome of an INDSCAL analysis is an overall group perceptual space reflecting the perceptual structure of the stimulus set, as well as a dimensional weight vector for each subject indicating the relative saliency of each dimension for that subject. Each of the 16 stimuli are represented as points in the perceptual space defined by the distinctive features, and the saliency weights for each subject reflect the degree to which that subject used each dimension in making his similarity judgments.

The present method offers at least two advantages over that used by Webster et al. (1973). First, the INDSCAL analysis provides precise quantitative estimates of featural saliency. Second, the INDSCAL method will permit individual subject differences to be considered.

\section{METHOD}

\section{Subjects}

Subjects were 34 undergraduate student volunteers at The Catholic University who were paid $\$ 3$ for their participation in a 1-h session. A random selection of 17 subjects was screened for hearing abnormalities by the Catholic University Speech and Hearing Clinic.

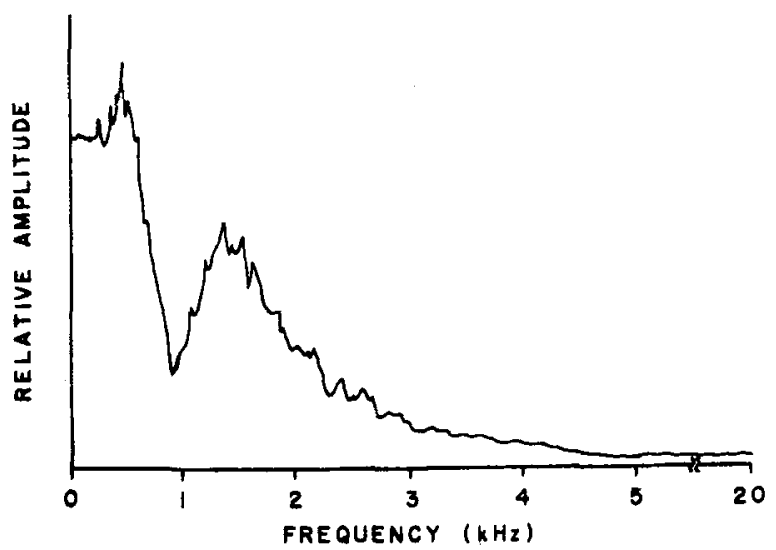

Figure 1. Spectral analysis of filter output with two formants $(600$ and $1,550 \mathrm{~Hz}$ ) while white noise $(20 \mathrm{~Hz}$ to $20 \mathrm{kHz})$ was applied at the input.

\section{Apparatus}

Four infinite-gain, state-variable, second-order filters were constructed (Graeme, 1971) and tuned to preselected formant frequencies of $600,940,1.550$. and $2.440 \mathrm{~Hz}$. The roll-off, formant $Q$ values and formant frequencies were calibrated to approximate those values reported by Webster et al. (1973). The output of each filter and combinations of various filters were summed by a variable gain amplifier. Individual filters or summed combinations were excited by $90-$ or $140-\mathrm{Hz}$ fundamental, square, or triangular wave signals. The response of each filter was investigated by analyzing its spectral composition with a Federal Scientitic Analyzer. Model UA-10. The response of a filter with two summed formants at 600 and $1.550-\mathrm{Hz}$ center frequencies is shown in Figure 1 (füter excited with broadband white noise. $20.20 \mathrm{~K} \mathrm{~Hz}$ ). A schematic representation of the spectra of each of the 16 sounds is presented in Figure 2.

Each of the 16 possible signals ( 2 driving frequencies $\times 2$ waveforms $x$ high or low frequency $x 1$ or 2 formants) was recorded onto magnetic tape with a Sony Model TC-270 tape recorder. Signal intensity was equated for RMS amplitude (Hewlett-Packard $400 \mathrm{H}$ True RMS Meter). Signal pairs were made by splicing 3-sec segments of these signals between $1-s e c$ segments of blank tape. Individual stimulus pairs were separated by a 4 -sec silent response period. A total of 120 randomized pairs of the 16 signals were generated. with each signal occurring with every other signal exactly

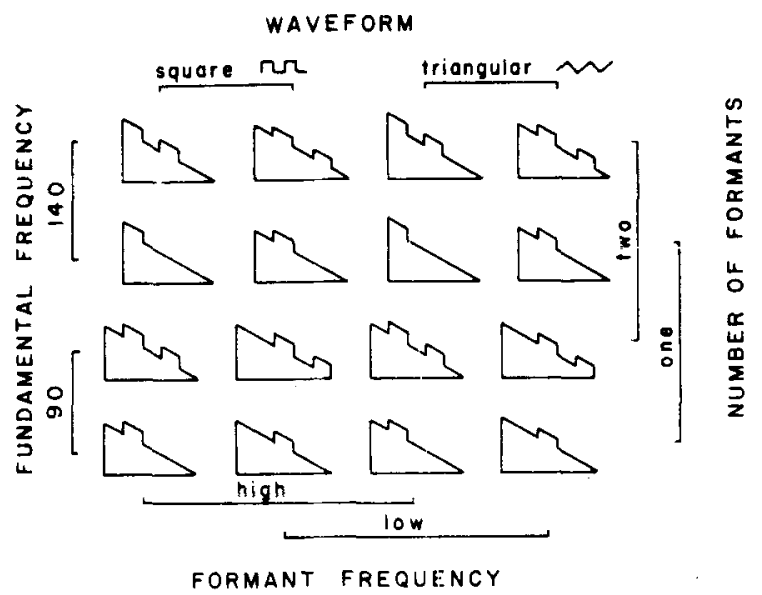

Figure 2. Schematic power spectra for each of the 16 sounds (adapted from Webster et al., 1973). 


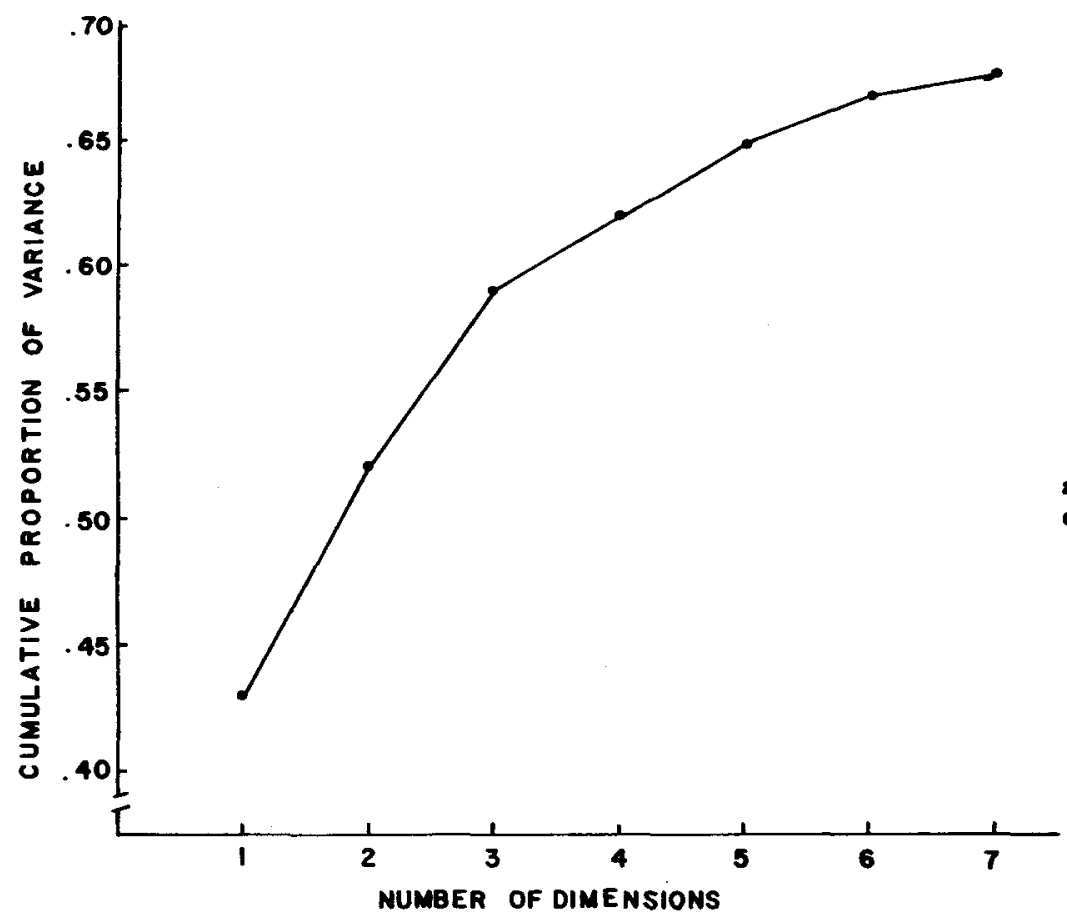

Figure 3. Total proportion of variance accounted for as a function of solution dimensionality.

once. The presentation order of stimuli within each pair was randomly determined to avoid any systematic time-order errors. A single stimulus tape was used for all subjects.

\section{Procedure}

Stimuli were presented on a Sony Model TC-270 tape recorder over a loudspeaker placed approximately $1.5 \mathrm{~m}$ in front of the subject. Amplifier gain was set to a comfortable listening level (approximately $65 \mathrm{~dB}$ re: .0002 dynes $/ \mathrm{cm}^{2}$ ). The use of a loudspeaker enabled more than one subject to be tested at a time, although no more than three subjects were tested during any one session. The subjects sat in a carpeted, sound-attenuated room and were provided with an answer sheet for manually recording their responses. Subjects were read instructions that described the nature of their task, and a 5-point rating scale for judging stimulus similarity $(1=$ very dissimilar, $5=$ very similar $)$ was explained to them. Examples of each of the 16 sounds were presented during a 5-min practice session to permit subjects to become accustomed to use of the scale. Following the practice session, subjects were asked to rate the degree of similarity of each of the 120 signal pairs by marking the appropriate box on their answer sheets. At the conclusion of the session, subjects were asked to complete a biographical information sheet and questionnaire. The data were analyzed on the Catholic University DECSystem 10 computer.

\section{RESULTS}

\section{Scaling Analysis}

An off-diagonal 16 by 16 matrix of similarity judgments was obtained for each of the subjects in the experiment. These data were subjected to a three-way scaling analysis using the INDSCAL procedure. Preliminary solutions were obtained in spaces of from one to seven dimensions. The total proportion of variance accounted for by each solution is presented in Figure 3. Although the total variance accounted for ranged from $43 \%$ with one dimension to $68 \%$ with seven dimensions, it is clear from Figure 2 that little additional variance is accounted for by increasing the dimensionality of the solution beyond three dimensions. Since three dimensions are also relatively easy to conceptualize and interpret, the threedimensional solution was selected for further analysis. ${ }^{1}$ The resulting normalized three-dimensional solution is represented graphically in Figure 4 and the coordinates for each of the 16 sounds are presented in Table 1. Visual inspection of these data suggests a correspondence between the psychological features $\psi_{1}$ and $\psi_{2}$ obtained in the solution and the physical

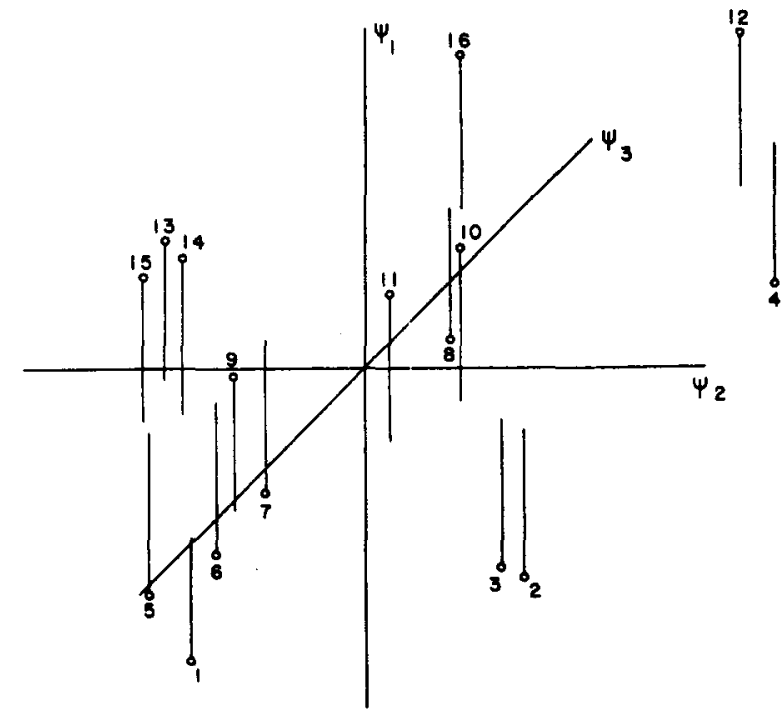

Figure 4. Normalized three-dimensional INDSCAL solution. 
Table 1

Stimulus Coordinates for Each of the 16 Auditory Signals

\begin{tabular}{|c|c|c|c|c|c|c|c|}
\hline \multirow[b]{2}{*}{ Signal } & \multicolumn{4}{|c|}{$\begin{array}{c}\text { Physical } \\
\text { Characteristics }\end{array}$} & \multicolumn{3}{|c|}{$\begin{array}{c}\text { Psychological } \\
\text { Dimensions }\end{array}$} \\
\hline & $\phi_{1}$ & $\phi_{2}$ & $\phi_{3}$ & $\phi_{4}$ & $\psi_{1}$ & $\psi_{2}$ & $\psi_{3}$ \\
\hline 1 & 90 & $\mathrm{Sq}$ & 1 & Lo & -.208 & -.002 & .377 \\
\hline 2 & 90 & $\mathrm{Sq}$ & 2 & Lo & -.261 & .382 & .139 \\
\hline 3 & 90 & $\mathrm{Sq}$ & 1 & $\mathbf{H i}$ & -.256 & .329 & .114 \\
\hline 4 & 90 & $\mathrm{Sq}$ & 2 & $\mathrm{Hi}$ & -.238 & .328 & -.486 \\
\hline 5 & 90 & $\mathrm{Tr}$ & 1 & Lo & -.278 & -.265 & .143 \\
\hline 6 & 90 & $\mathrm{Tr}$ & 2 & Lo & -.263 & -.204 & .079 \\
\hline 7 & 90 & $\mathrm{Tr}$ & 1 & $\mathrm{Hi}$ & -.258 & -.223 & -.058 \\
\hline 8 & 90 & $\mathrm{Tr}$ & 2 & $\mathrm{Hi}$ & -.233 & -.134 & -.349 \\
\hline 9 & 140 & $\mathrm{Sq}$ & 1 & Lo & .232 & .020 & .318 \\
\hline 10 & 140 & $\mathrm{Sq}$ & 2 & Lo & .262 & .229 & .074 \\
\hline 11 & 140 & $\mathrm{Sq}$ & 1 & $\mathrm{Hi}$ & .228 & .166 & .167 \\
\hline 12 & 140 & $\mathrm{Sq}$ & 2 & $\mathrm{Hi}$ & .265 & .342 & -.397 \\
\hline 13 & 140 & $\mathrm{Tr}$ & 1 & Lo & .238 & -.330 & .023 \\
\hline 14 & 140 & $\mathrm{Tr}$ & 2 & Lo & .272 & -.241 & .087 \\
\hline 15 & 140 & $\operatorname{Tr}$ & 1 & $\mathrm{Hi}$ & .250 & -.287 & .120 \\
\hline 16 & 140 & $\mathrm{Tr}_{\mathrm{r}}$ & 2 & $\mathrm{Hi}$ & .249 & -.109 & -.352 \\
\hline
\end{tabular}

Note $-\phi_{1}=$ fundamental frequency, $\phi_{2}=$ waveform, $\phi_{3}=$ number of formants, $\phi_{4}=$ formant frequency: $L o=600$ and 1550 or $600 \mathrm{~Hz}$ and $\mathrm{Hi}=940$ and 2440 or $940 \mathrm{~Hz}$.

Table 2

Correlations Between Physical Characteristics and Psychological Features

\begin{tabular}{|c|c|c|c|}
\hline \multirow{2}{*}{$\begin{array}{c}\text { Physical } \\
\text { Characteristic }\end{array}$} & \multicolumn{3}{|c|}{ Psychological Feature } \\
\hline & $\psi_{1}$ & $\psi_{2}$ & $\psi_{3}$ \\
\hline Fundamental & $.99 *$ & .11 & .02 \\
\hline Waveform & .01 & $.90^{*}$ & .15 \\
\hline Formant Frequency & .00 & .21 & $.62 * *$ \\
\hline Number of Formants & .03 & .30 & $.61^{* *}$ \\
\hline${ }^{*} p<.001$ & & ${ }^{*} p<$ & \\
\hline
\end{tabular}

parameters fundamental frequency and waveform, respectively. Sounds having a low fundamental frequency $(90 \mathrm{~Hz})$ cluster with negative values along $\psi_{1}$, while sounds with a high fundamental $(140 \mathrm{~Hz})$ cluster with positive values. Similarly, square waves tend to have positive values and triangular waves tend to have negative values along $\psi_{2}$. The interpretation of the third dimension, $\psi_{3}$, is less obvious: Notice, however, that sounds having two high-frequency formants ( 940 and $1,440 \mathrm{~Hz}$ ), signals $\mathrm{S}_{4}, \mathrm{~S}_{8}, \mathrm{~S}_{12}$, and $S_{16}$, tend to be distinguished from the remaining sounds along this dimension. This suggests that the third dimension reflects a combined function of the two formant parameters.

These observations were confirmed in a statistical analysis of the scaling solution. Point-biserial correlation coefficients were computed between each of the four physical characteristics and the three psychological features. The results of this analysis appear in Table 2. The two source parameters, fundamental frequency and waveform, correlated significantly with $\psi_{1}$ and $\psi_{2}$, respectively, and, as anticipated above, both formant parameters correlated highly with dimension $\psi_{3}$. No other correlations reached significance at the .05 level. In summary, it appears that $\psi_{1}$ corresponds in a direct way to fundamental frequency, $\psi_{2}$ to the signal waveform. and $\psi_{3}$ to a combined function of the two formant parameters.

\section{Hierarchical Clustering Analysis}

Shepard (1972) has suggested that a multidimensional scaling analysis combined with an independent hierarchical clustering analysis would lead to more complete understanding of the data. If the features obtained from the scaling analysis accurately reflect the subject's underlying perceptual structure, then the hierarchical structure revealed by the clustering analysis should be consistent with the scaling solution.

A mean, off-diagonal similarity matrix was obtained for the present stimuli by collapsing across individual subject matrices. These data were subjected to Johnson's hierarchical clustering analysis/connectedness method (Johnson, 1967). The results of this analysis are displayed in Figure 5, and the obtained clusters are displayed embedded in the multidimensional scaling solution for lowfundamental $\left(\mathrm{S}_{1}-\mathrm{S}_{8}\right)$ and high-fundamental $\left(\mathrm{S}_{9}-\mathrm{S}_{16}\right)$ sounds in Figures $6 \mathrm{a}$ and $6 \mathrm{~b}$, respectively. Specific stimuli are grouped according to the hierarchical structure indicated in the clustering analysis. Notice that the clustering and scaling analyses produced nearly identical structural descriptions of these datain no case do the stimulus clusters overlap in the perceptual space. This observed consistency lends further support to the perceptual structure revealed by the scaling analyses.

\section{Individual Differences Analysis}

As indicated above, the INDSCAL model preserves individual subject information while extracting the underlying group perceptual space. Since complex nonspeech sounds differ widely in importance for different individuals (e.g., certain sounds and cues are extremely important for musicians and sonarmen), it is likely that large individual differences

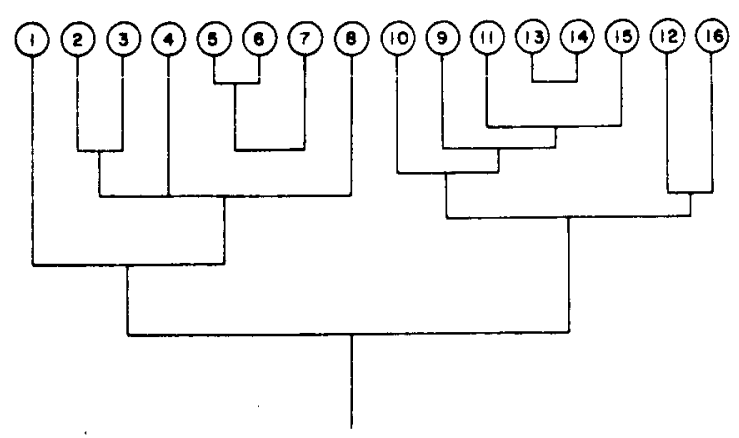

Figure 5. Stimulus clusterings produced by Johnson's hierarchical clustering analysis/connectedness method. 


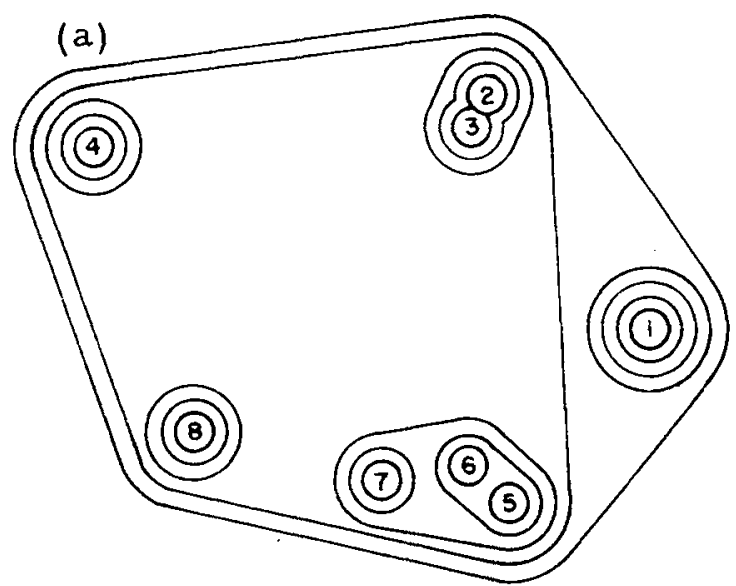

(b)

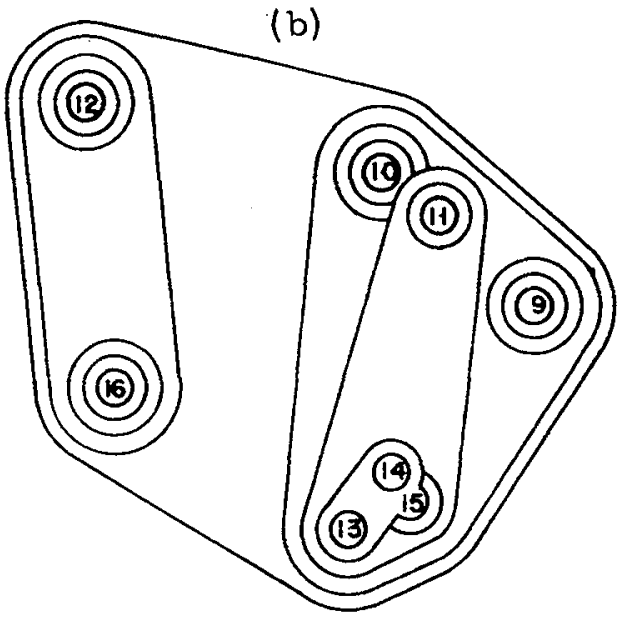

Figure 6. (a) Representation of hierarchical clustering analysis embedded in the INDSCAL solution for $90-\mathrm{Hz}$ signals. (b) Representation of hierarchical clustering analysis embedded in the INDSCAL solution for $140-\mathrm{Hz}$ signals.

would occur in the perception of these sounds. Individual differences were considered in the present data by examining the dimensional saliency or weighting factors for the three psychological features for each of the 34 subjects. The individual feature saliencies for each of the three dimensions are presented in Table 3. Notice that, while many subjects (e.g., Subjects 5, 6, 7) relied almost exclusively on the $\psi_{1}$ dimension, some subjects (e.g.. Subjects $20,25,34)$ used $\psi_{2}$ and $\psi_{3}$ to an equal or greater degree.

This finding raises the possibility that the group scaling solution discussed above is only partially representative of an individual subject's perceptual structure. In other words, the three psychological dimensions reported above appear to be distinctive features that subjects may use in perceiving these sounds, but any particular subject is likely to emphasize only some subset of these features. This possibility was examined further by computing Pearson product-moment correlations between the individual subject saliency weights for each of the psychological dimensions. A high positive correlation between the weights on two dimensions would indicate that the dimensions tend to be emphasized together. That is, subjects emphasizing (or de-emphasizing) the one dimension would also tend to emphasize (or de-emphasize) the other dimension. On the other hand, a high negative correlation between the saliency weights for two features would suggest a different dimensional independence: Subjects stressing the one dimension would tend to de-emphasize the ofther, and vice versa. The results of this analysis appear in Table 4a. It is clear from this analysis that a significant negative relation exists between $\psi_{1}$ (fundamental) and the remaining dimensions, $\psi_{2}$ (waveform) and $\psi_{3}$ (formants), while $\psi_{2}$ and $\psi_{3}$ are positively related. These results suggest that there are at least two types of individual subjects: those who emphasize $\psi_{1}$ (fundamental) and de-emphasize $\psi_{2}$ (waveform) and $\psi_{3}$ (formants), and those who emphasize $\psi_{2}$ and $\psi_{3}$ and de-emphasize $\psi_{1}$.

This finding was examined further by dividing the

Table 3

Individual Subject (S) Saliency Weights for Each Feature

\begin{tabular}{llllllll}
\hline & \multicolumn{3}{c}{ Saliency Weight } & \multicolumn{4}{c}{ Saliency Weight } \\
$\mathrm{S}$ & $\mathrm{W}_{1}$ & $\mathrm{~W}_{2}$ & $\mathrm{~W}_{3}$ & $\mathrm{~S}$ & $\mathrm{~W}_{1}$ & $\mathrm{~W}_{2}$ & $\mathrm{~W}_{3}$ \\
\hline $1^{*}$ & .752 & .238 & .235 & 18 & .893 & .137 & .069 \\
2 & .458 & .226 & .464 & 19 & .385 & .052 & .093 \\
$3^{*}$ & .632 & .178 & .234 & $20^{*}$ & .382 & .248 & .446 \\
$4^{*}$ & .400 & .002 & .213 & $21^{*}$ & .597 & .440 & .255 \\
$5^{*}$ & .900 & .140 & .081 & $22^{*}$ & .888 & .049 & .125 \\
$6^{*}$ & .819 & .052 & .263 & 23 & .778 & .232 & .253 \\
$7^{*}$ & .923 & .097 & .114 & 24 & .706 & .258 & .295 \\
8 & .229 & .626 & .314 & 25 & .217 & .464 & .366 \\
9 & .768 & .003 & .163 & 26 & .935 & .070 & .106 \\
$10^{*}$ & .912 & .175 & .162 & 27 & .837 & .080 & .260 \\
11 & .281 & .569 & .371 & $28 *$ & .588 & .274 & .250 \\
12 & .755 & .210 & .343 & 29 & .451 & .402 & .421 \\
13 & .709 & .194 & .302 & 30 & .742 & .256 & .330 \\
14 & .325 & .302 & .299 & 31 & .873 & .075 & .131 \\
$15^{*}$ & .199 & .574 & .449 & 32 & .393 & .507 & .291 \\
16 & .742 & .204 & .248 & 33 & .675 & .224 & .277 \\
17 & .232 & .213 & .140 & 34 & .222 & .421 & .186 \\
\hline
\end{tabular}

Note- * indicates musically experienced subjects.

Table 4

Cross Correlations Between Individual Subject Saliency Weights

Saliency Weight $\quad W_{1}{ }^{\text {Saliency Weight }} \mathrm{W}_{2}$

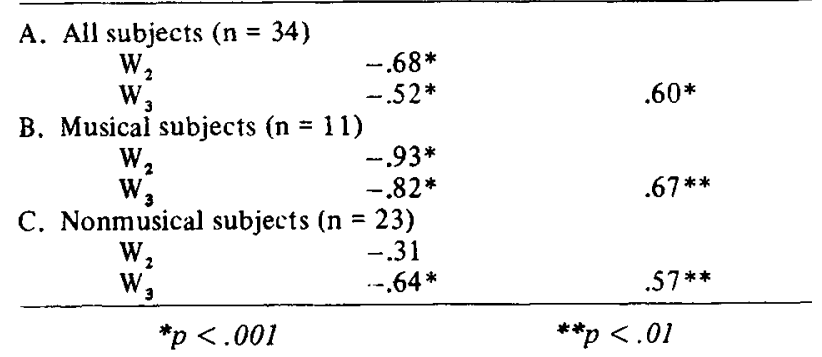


subjects post hoc into musically sophisticated and musically naive groups according to their responses on a postexperimental questionnaire. A musically sophisticated subject was defined to be anyone who had played any musical instrument for a period greater than 3 years, while a musically naive subject had less experience with a musical instrument. The above saliency correlations were recomputed on the separate groups. These data are presented in Tables $4 \mathrm{~b}$ and $4 \mathrm{c}$ for the musical $(\mathrm{n}=11)$ and nonmusical ( $\mathrm{n}$ $=23$ ) subjects, respectively. The results parallel the group findings; however, the correlations are somewhat larger for the musical subjects than for the nonmusical subjects. This suggests that the musically sophisticated subjects are more homogeneous with regard to feature saliency than the nonmusical subjects-as a group, the musical subjects are more likely to stress $\psi_{1}$ and de-emphasize $\psi_{2}$ and $\psi_{3}$ than are the nonmusical subjects.

These differences are evident in the plot of the individual subject saliency space for the $\psi_{1}$ (fundamental) and $\psi_{3}$ (formants) dimensions displayed in Figure 7. Each of the 34 subjects is represented as a point in the space, indexed by their saliency weights for the two dimensions shown. The distinction between musical and nonmusical subjects is obvious in the figure. Musical subjects tend to cluster with high saliences for $\psi_{1}$ and low saliences for $\psi_{3}$, while the nonmusical subjects are distributed across the full range of saliency values. Similar plots for the $\psi_{1} / \psi_{2}$ and $\psi_{2} / \psi_{3}$ dimensions may be obtained from the saliency weights in Table 3.

In summary, an analysis of individual subject data in the present study indicated that subjects vary widely in the distinctive features they emphasize in perceiving complex sounds. It was found that some subjects tended to emphasize $\psi_{1}$ (fundamental) exclusive of the other dimensions while other subjects stressed $\psi_{2}$ and $\psi_{3}$ exclusive of $\psi_{1}$. A breakdown of subjects into musically sophisticated and musically naive groups indicated that most musical subjects fell into the former category, while nonmusical subjects were divided between the two categories.

\section{DISCUSSION}

It is clear from the above analysis that multidimensional scaling provides a useful technique for determining the distinctive features involved in the perception of complex nonspeech sounds. As indicated in the introduction, multidimensional scaling procedures have proven helpful in the analysis of human speech perception, but the methods have not been systematically applied to auditory perception in the nonspeech realm (e.g., Wish \& Carroll, 1974a). In the present study, much of the total variance in the subject's similarity judgments was accounted for by an interpretable, three-dimensional scaling solution.

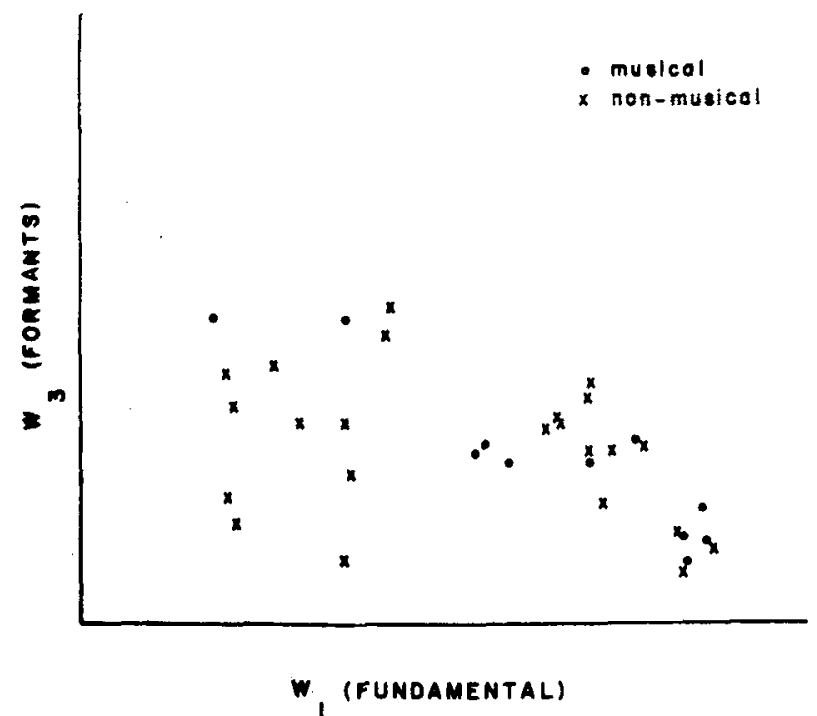

Figure 7. Representation of individual subjects in a two-dimensional salkency space.

The three dimensions have been viewed as corresponding to the psychological characteristics or distinctive features that subjects used in perceiving the 16 stimuli used in the present study. A correspondence was observed between each of the psychological dimensions obtained in the scaling solution and the physical parameters varied to generate the 16 sounds. The $\psi_{1}$ dimension corresponds to fundamental frequency, $\psi_{2}$ to waveform, and $\psi_{3}$ to a combination of the two formant parameters.

We can speculate further that $\psi_{1}$ may reflect the common auditory dimension of pitch. It is likely, however, that the formant dimension, $\psi_{3}$, also contributes to the subject's perception of pitch, but in a manner that is independent of fundamental frequency. This argument is supported by the finding that $\psi_{3}$ tends to separate sounds according to maximum formant frequency, and in particular, to separate the four sounds having a formant centered at the highest frequency (i.e., $2,440 \mathrm{~Hz}$ ) from the remaining sounds. These findings are consistent with the argument that pitch perception depends on the spectral composition as well as on the fundamental frequency of an auditory signal (e.g., Plomp, 1967).

The high correlation between the second psychological dimension, $\psi_{2}$, and the waveform parameter (i.e., square vs. triangle) suggests a straight-forward interpretation. The differences in spectral composition of square and triangular signals lead to a perceptual difference in tonal quality similar to that occurring in the sounds produced by different musical instruments. These perceptual differences are well documented empirically. However, in the present study, there is evidence that the $\psi_{2}$ dimension may 
reflect signal loudness in addition to tonal quality. In generating the stimuli for the present study, stimuli were equated for RMS amplitude rather than for loudness or perceived intensity. ${ }^{2}$ As a result, the square-wave signals had greater energy than the triangular signals-a difference likely to produce differences in perceived intensity.

In order to examine the possible role of signal loudness, the subjects were asked to rate the loudness of each of the 16 sounds on a scale of 1 (very soft) to 5 (very loud) immediately after completing their comparative judgments. A mean loudness rating was computed for each stimulus, and these data were correlated with each of the psychological dimensions obtained in the scaling solution. This analysis revealed Pearson product-moment correlations of .15 , .87 , and .07 between the mean loudness ratings and the features $\psi_{1}, \psi_{2}$, and $\psi_{3}$, respectively. Only the correlation with $\psi_{2}$ reached statistical significance $[\mathrm{t}(14)=7.73, \mathrm{p}<.001]$. This finding confirms the above speculation that the $\psi_{2}$ (waveform) dimension corresponds closely to loudness, and that the other two dimensions, $\psi_{1}$ (fundamental) and $\psi_{3}$ (formants), were independent of subjective intensity.

The 16 complex sounds examined in the present study were similar to those used by Webster et al. (1973) in an auditory classification task. In Webster's study, subjects were required to learn a classification for each of the 16 sounds using an arbitrary set of labels. Webster et al, attempted to determine the relative psychological importance of the four physical parameters by examining the confusion matrices generated by subjects during a 2-week learning period. Although the conclusion reached on the basis of this analysis roughly paralleled the findings of the present study, no precise quantitative estimates of featural saliency were provided. Therefore, in order to obtain a more precise comparison, an interstimulus similarity matrix was established from the Euclidean interstimulus distances in the three-dimensional stimulus space, and compared with the overall confusion matrix reported by Webster et al. A statistically reliable correlation was obtained $[\mathrm{r}=$ $-.53, \mathrm{t}(118)=-6.78, \mathrm{p}<.001]$. While the overall fit is not impressive, stimulus, subject, and task differences between the two studies would all serve to increase variability. In addition, Shepard (1972) has pointed out that the psychological processes involved in classification and similarity comparison tasks are likely to involve different stimulus characteristics and would therefore lead to different underlying perceptual structures. In general, however, the present findings compare favorably with Webster's.

The three-dimensional scaling solution described above accounts for approximately $59 \%$ of the total variance in the subjects' similarity ratings. While the overall variance accounted for appears small relative to the results reported for similar work with speech (e.g., Shepard, 1972, accounts for $99 \%$ with three dimensions), the difference is not surprising in light of the differences between speech and the present nonspeech stimuli. First, the stimuli used in the present study were highly similar perceptually, resulting in relatively poor signal discriminability. In addition, the distinctive features involved in human speech perception are determined by socio-cultural and physiological (e.g., Eimas \& Corbit, 1973) factors which, for obvious reasons, are uniform across individuals. In contrast, individuals need not extract precisely the same features in processing complex nonspeech sounds. As a result, the three-dimensional solution examined above was not adequate for all subjects, sincei it is likely that some subjects were using stimulus features not represented in the solution.

The present data revealed substantial individual differences in the features emphasized. Some subjects-especially musically sophisticated subjects -relied primarily on $\psi_{1}$ (fundamental), while others relied primarily on $\psi_{2}$ (waveform) and $\psi_{3}$ (formants). Such differences may result from an attentional process (e.g., Kinchla, 1973) that selectively enhances one or another of the distinctive features in the complex signal. For example, in turning a musical instrument, it is necessary to ignore the spectral characteristics of the sound (i.e., the instrument producing the sound) and attend to the fundamental of the sound (i.e., the note being played). Musically sophisticated subjects appear to be better at selectively attending to the most salient distinctive feature, $\psi_{1}$ (fundamental), than musically naive subjects. The issue of why subjects, musically experienced or not, would base similarity ratings primarily on pitch is an open question.

In conclusion, the present study has demonstrated the utility of multidimensional scaling methods in the analysis of complex auditory perception in the nonspeech mode. The methods may be generally useful in further clarifying the relation between various physical characteristics of complex sounds and their perceptual correlates. Such analyses can be of considerable heuristic and theoretical value for research on auditory perception. For example, the role of attentional mechanisms in determining featural saliency and the influence of experience on these mechanisms remain to be determined.

\section{REFERENCE NOTES}

1. Wish, M. An INDSCAL analysis of the Miller-Nicely consonant confusion data. Paper presented at meetings of the Acoustical Society of America, Houston, November 1970.

2. Carterette, E., \& Barnebey, A. Recognition memory for voices. Paper read to the Psychonomic Society, Boston, Massachusetts, November 1974. 


\section{REFERENCES}

Carrole, J. D., \& Chang, J. J. Analysis of individual differences in multidimensional scaling via an $\mathrm{N}$-way generalization of Eckart-Young decomposition. Psychometrika, 1970, 35, 283-319.

Carroll, J. D., \& Wish, W. Multidimensional perceptual models and measurement methods. In E. Carterette \& M. Friedman, M. (Eds.), Handbook of perception (Vol. II). New York: Academic Press, 1974. (a)

CARroll. J. D., \& Wish, M. Models and methods for three-way multidimensional scaling. In D. H. Krantz, R. C. Atkinson, R. D. Luce, \& P. Suppes (Eds.), Measurement, psychophysics, and neural information processing (Vol. II). San Francisco: Freeman, 1974. (b)

Eimas. P. D., \& Corbit, J. D. Selective adaptation of linguistic feature detectors. Cognitive Psychology, 1973, 4, 99-109.

GARNER. W. R. The processing of information and structure. Potomac, Md: Erlbaum, 1974.

GraEme, J. G. (Ed.) Operational amplifiers: Design and applications. New York: McGraw-Hill, 1971.

Johnson, S. C. Hierarchical clustering schemes. Psychometrika, $1967,32,241 \cdot 254$.

Kavanagh, J. F., \& Mattingly, I. G. Language by ear and by eye. Cambridge: M.I.T. Press, 1972.

Kinchla, R. A. Selective processes in sensory memory: A probe-comparison procedure. In S. Kornblum (Ed.), Attention and performance (Vol. IV). New York: Academic Press, 1973.

LIBERMAN, A. M. The grammars of speech and language. Cognitive Psychology, 1970, 1, 303-323.

Massaro, D. W. Preperceptual images, processing time, and perceptual units in auditory perception. Psychological Review, 1972, 79. 124-145.

Mattingly, I. G., Liberman, A. M., Syrdal, A. K., \& Halwes, T. Discrimination in speech and nonspeech modes. Cognitive Psychology, 1971, 2, 131-157.

Miller, G. A., \& Nicely, P. E. An analysis of perceptual confusions among some English consonants. Joumal of the Acoustical Society of America, 1955, 27, 338-352.
Norman, D. A., \& Bobrow, D. G. On data-limited and resource-limited processes. Cognitive Psychology, 1975, 7, 44-64.

Plomp, R. Pitch of complex tones. Journal of the Acoustical Society of America, 1967, 41, 1526-1533.

Shepard, R. N. Psychological representation of speech sounds. In E. David \& P. Denes (Eds.), Human communication: A unified view. New York: McGraw-Hill, 1972.

Shepard, R. N., Nerlove, S., \& Romney, S. (Eds.) Multidimensional scaling theory and applications in the behavioral sciences (Vol. I). New York: Seminar Press, 1972.

TORGERson, W. S. Theory and methods of scaling. New York: Wiley, 1958.

Webster, J. C., Woodhead, M. M., \& Carpenter, A. Perceptual confusions between four-dimensional sounds. Journal of the Acoustical Society of America, 1973, 53, 448-456.

WISH, M., \& CARROLL, J. D. Applications of individual differences scaling to studies of human perception and judgment. In E. Carterette \& M. Friedman (Eds.), Handbook of perception (Vol. II). New York: Academic Press, 1974.

\section{NOTES}

1. Because of the four-dimensional physical structure of the stimulus set. the four-dimensional scaling solution was examined in considerable detail. This analysis revealed that the first three psychological dimensions in the four-dimensional solution corresponded to the dimensions in the three-dimensional solution. The additional, fourth, dimension appeared to reflect the number of formants parameter, however, the correspondence was tenuous at best (the point-biserial correlation between the coordinates of this dimension and number of formants was .40). Consequently, the three-dimensional solution was accepted as providing the most parsimonious estimate of the underlying perceptual structure.

2. This procedure was followed in an attempt to approximate the signals used by Webster et al. (1973) as closely as possible.

(Received for publication June 27, 1975; revision received September 15, 1975.) 